La notion de temps:

temps physique et relativité, la dynamique du point matériel. Par Ernest Esclangon. Pp. iv + 77. (Paris : Gauthier-Villars, 1938.) 20 francs.

7 HERE is nothing very new in this little essay by the Director of the Paris Observatory; but it is a lucidly written contribution to that subject which is now almost universally known as the philosophy of science. M. Esclangon introduces his discussion by a consideration of metaphysical time, which he decides can be reduced to a sensation that is individual in every one of us, and owes its existence to a kind of biological clock to which we refer our sensory perceptions. The extension of this idea to a Being who, although infinite and therefore beyond our powers of conception, yet dominates the universe and is equipped with a form of consciousness akin to our own, has given birth to the illusion of a real and absolutely independent time. This illusion has only been dispelled-with considerable difficulty -from human minds by the concept of relativity. M. Esclangon concludes that, apart from the various 'times' defined by a series of conventions which are the creation of science and form the subject of dis. cussion of the rest of the book, there is only this subjective, individual time, of which the true nature must for ever remain mysterious and impenetrable.

For the rest, M. Esclangon discusses these various scientific times-of which one example is the 'time' of the special theory of relativity-examines the principle of reciprocity with a description of Fizeau's experiment, the invariance of natural laws in uniform translation and the equations of dynamics which are deduced from the principle of reciprocity. $\mathrm{He}$ does not minimize the difficulties involved in all physical theory, and is of the opinion that at present no firm territory is to be found in those regions which lie at the frontiers of present-day science. A. v. Z.

Bird Flocks and the Breeding Cycle:

a Contribution to the Study of Avian Sociality. By Dr. F. Fraser Darling. Pp. $x+124+1$ plate. (Cambridge: At the University Press, 1938.) 6s. net.

7 HE author spent about two years on uninhabited 1 Priest Island, off the north-west coast of Scotland. There he observed the breeding behaviour of the sea-birds, particularly of the lesser black-backed and herring gulls. He reports on his observations in this book, and presents a shrewd conclusion which one feels may lead to the solution of many problems, such as the lack of elasticity or recuperative power in the population of a species when its numbers fall below a certain level. It is understood by the majority of naturalists that the breeding behaviour of individual pairs is a sufficient stimulus to bring mating to its successful conclusion. Now Dr. Darling finds in certain birds which have social habits that the proper pitch of emotion can only be reached by the combined behaviour of a certain minimum population.

This is an important contribution to the study of animal behaviour, and a book which brings to the layman from the authority himself the result of his scientific observation.
The Hypothalamus :

Morphological, Functional, Clinical and Surgical Aspects. By Dr. W. Le Gros Clark, Dr. John Beattie, Dr. George Riddoch and Norman M. Dott. (The Henderson Trust Lectures, Nos. 13-16). Pp. xii + 212. (Edinburgh and London: Oliver and Boyd, 1938.) 12s. $6 d$. net.

THE importance of the region of the brain near the pituitary gland has been recognized with increasing clearness in recent years, and a review of modern knowledge of the hypothalamus is therefore welcome. The book consists of an amplification of the Henderson Trust Lectures which were delivered in Edinburgh in October 1936 by the four authors. Prof. Le Gros Clark's section gives a detailed account of the development and anatomy of the hypothalamus in mammals, with a special section on man and smaller sections on reptiles, amphibians and fishes. Dr. Beattie discusses the physiological evidence which has led to the conclusions that the hypothalamus controls sleep, the pituitary and the reactions of the body to heat and cold, and that the anterior portion controls the parasympathetic system and the posterior portion the sympathetic system. Dr. George Riddoch discusses the pathology of the region and Mr. Norman Dott describes four cases in which he removed tumours. Both of them independently describe the clinical symptoms and signs associated with hypothalamic disease--the hunger and the thirst, the obesity and the cachexia, and the disturbances of sleep, sex, temperature, and the stomach.

The book is attractively produced with more than a hundred figures and diagrams and an adequate bibliography.

\section{About Petroleum}

By J. G. Crowther. Pp. xiv +181. (London: Oxford University Press, 1938.) 7s. 6d. net.

7 HERE are a great many people who use petroleum in some form or other every day of their lives without really knowing anything about it. Some of them from time to time are actuated by the desire to find out what it is and where it comes from. Unfortunately, however, petroleum is such a complicated substance, both chemically and physically, that the normal run of text-books is comprehensible only to those who have specialized knowledge of the subject. Mr. J. G. Crowther in his book anticipates questions that must arise in the minds of motor drivers, business men, housewives and others in the course of their daily duties, and endeavours to give simple answers to these. The result is that within the confines of a slim volume information is given in a form palatable to the non-scientific mind on all major aspects of petroleum technology. The text is amplified by means of carefully selected photographs and easily interpreted diagrams. Moreover, there is an exhaustive index which facilitates easy reference to the vast number of facts which have been used as a basis of this work. There is little doubt that the author's aim will be achieved and that this book will serve as an excellent primer to more comprehensive treatises on petroleum. 